\title{
A Curious Case of Acute Glomerulonephritis - Staphylococcus vs Lupus: Case Report and Literature Review
}

\author{
Maham A. Mehmood ${ }^{1}$, Hitesh Gurjar ${ }^{1}$, Tegveer Sindhu ${ }^{1}$, Asim Haider ${ }^{1}$, Mahnoor Arshad ${ }^{2}$ \\ 1. Internal Medicine, BronxCare Health System, New York, USA 2. Internal Medicine, Faisalabad Medical University, \\ Faisalabad, PAK
}

Corresponding author: Maham A. Mehmood, mahum.arshad89@gmail.com

\begin{abstract}
Here we report a case of a 65 -year-old female, where we encountered acute glomerulonephritis. The patient initially presented with a hemorrhagic blister involving the second through fourth toes with serosanguinous discharge and was on hydralazine for blood pressure control. Differentials included Staphylococcusassociated/lupus nephritis/anti-neutrophil cytoplasmic autoantibody (ANCA) vasculitis. Still, detailed history and meticulous clinical approach with supporting labs and imaging helped us to narrow it to Staphylococcus-associated glomerulonephritis, which is rarely encountered in clinical practice and is associated with high mortality. The management of the patient resulted in a positive outcome and she was discharged home.
\end{abstract}

Categories: Infectious Disease, Rheumatology

Keywords: staphylococcus, lupus, hydralazine, kidney biopsy, methylprednisone

\section{Introduction}

Acute glomerulonephritis is often encountered in clinical practice. The differentials are broad and include lupus nephritis, vasculitis, infection-related, and immunoglobulin A (IgA) nephropathy. Good history, physical and meticulous laboratory investigations help narrow down the differentials, as prompt initiation of management can be critical in preventing morbidity and mortality. Staphylococcus-related glomerulonephritis is rare, and early recognition and eradication of infection can be life-saving.

Review began 01/21/2021 Review ended 02/02/2021 Published 02/04/2021

(c) Copyright 2021

Mehmood et al. This is an open access article distributed under the terms of the Creative Commons Attribution License CC-BY 4.0., which permits unrestricted use, distribution, and reproduction in any medium, provided the original author and source are credited.

\section{Case Presentation}

A 65-year-old female with medical comorbidities of dementia, diabetes (diet controlled), and hypertension (controlled on hydralazine) presented complaining of progressively worsening left foot ulcer for three days. As per the patient, the ulcer started as a blister and since worsened, accompanied by severe pain. On presentation, her blood pressure was $145 / 78 \mathrm{mmHg}$, pulse $71 / \mathrm{min}$, respiratory rate $14 / \mathrm{min}$, temperature $98 \mathrm{~F}$. On examination, she was at her baseline mental status (alert, oriented to self and place); her left foot was tender with a hemorrhagic blister involving the second through fourth toes with serosanguinous discharge. The rest of the physical examination was normal. Laboratory examination was noted to have acute kidney injury (blood urea nitrogen (BUN) and creatinine $24 \mathrm{mg} / \mathrm{l}$ and $2.4 \mathrm{mg} / \mathrm{l}$ respectively), baseline renal function was entirely normal six months prior to presentation, hemoglobin/hematocrit of $10 \mathrm{~g} / \mathrm{dl}$ and $32 \%$ respectively, white blood cell $7 \mathrm{k} / \mathrm{ul}$, erythrocyte sedimentation rate (ESR) $120 \mathrm{~mm} / \mathrm{hr}$, C-reactive protein 178 $\mathrm{mg} / \mathrm{l}$. Urine analysis was significant for large hematuria and trace proteinuria, urine protein $51(0-31 \mathrm{mg} / \mathrm{dl})$ and urine creatinine 60 (20-200 mg/dl) (Table 1). 


\section{Cureus}

\begin{tabular}{|c|c|c|}
\hline Labs & Patient result & Reference range \\
\hline Anti-peroxidase & $<1$ & $<1 \mathrm{Al}$ \\
\hline Anti Myeloperoxidase & 7.6 & $<1 \mathrm{Al}$ \\
\hline Anti-RNP & $<1$ & $<1 \mathrm{Al}$ \\
\hline Anti-Sm & $\begin{array}{lll}<1 & & \end{array}$ & $<1 \mathrm{Al}$ \\
\hline Glomerular basement membrane antibody & $<1$ & $<1 \mathrm{Al}$ \\
\hline Histone antibody & 9.6 & $<1 U$ \\
\hline Anti-DNA AB & 5 & $<4 \mid \mathrm{IU} / \mathrm{ml}$ \\
\hline HIV-1/2Ab (EIA) & Negative & Non-reactive \\
\hline RPR screen & Non-reactive & Non-reactive \\
\hline Anti-HCV & Negative & Negative \\
\hline
\end{tabular}

\section{TABLE 1: Autoimmune workup}

RPR: rapid plasma reagin, RNP: ribonucleoprotein, anti-Sm: anti-Smith, HCV: hepatitis C virus, EIA: enzyme immunoassay

X-ray chest was negative for any acute process. The patient was suspected of having clinical necrotizing fasciitis, she refused computed tomography (CT) of the lower extremity and underwent incision drainage, and debridement and cultures were sent. The patient was started on daptomycin/meropenem/clindamycin and was transferred to the medical floor. Kidney injury was presumably due to prerenal due to underlying infection, that was managed conservatively without dialysis. Antibiotics were deescalated to cefazolin/metronidazole as the cultures from the foot grew methicillin-sensitive Staphylococcus aureus. Her post-operative course was complicated with poor foot healing, and the patient ended up getting transmetatarsal amputation and then below-knee amputation (BKA) in a matter of days.

Histopathology later reported skin and subcutaneous tissue with acute and chronic inflammation, abscess and granulation tissue. Soft tissue margin and bone margin of resection were unremarkable.

The renal function continued to worsen, and the creatinine jumped to $4.4 \mathrm{mg} / \mathrm{l}$. The antinuclear antibody was positive with a titer of 1:320, hypocomplementemia (C3 was $53 \mathrm{mg} / \mathrm{dl}$ and C4 was $3 \mathrm{mg} / \mathrm{dl}$ ). At this point, differentials included post-inflammatory glomerulonephritis versus late-onset systemic lupus nephritis versus drug-induced lupus. Hydralazine was placed on hold. Other pertinent labs revealed positive antihistone antibodies 9.6 unit $($ ref $<0.1)$, cardiolipin IgM $>150$ (normal $<20$ ) and myeloperoxidase antibodies 9 units and 7.6 units respectively, creatinine jumped up to $6.6 \mathrm{mg} / \mathrm{l}$, anti-DNA, glomerular basement antibody, and antiphospholipid antibody were negative. Hepatitis panel including hepatitis B and C were negative. She also tested negative for HIV. Another differential of anti-neutrophil cytoplasmic autoantibody (ANCA)associated vasculitis was included, Rheumatology was taken on board, the patient was started on a pulsed dose of methylprednisone, and renal biopsy was planned to differentiate between lupus nephritis/pauciimmune glomerulonephritis. The patient's clinical course took a turn for the worse when she became hypoxic on the floor, was placed on the high flow oxygen, and transferred to the critical care unit. CT chest at this point revealed diffuse mixed interstitial and alveolar airspace disease and small pleural effusions (Figure 1). She also had a $1 \mathrm{~g}$ drop in hemoglobin from baseline requiring one packed red blood cell transfusion. She tested negative for coronavirus disease 2019 (COVID-19). 


\section{Cureus}

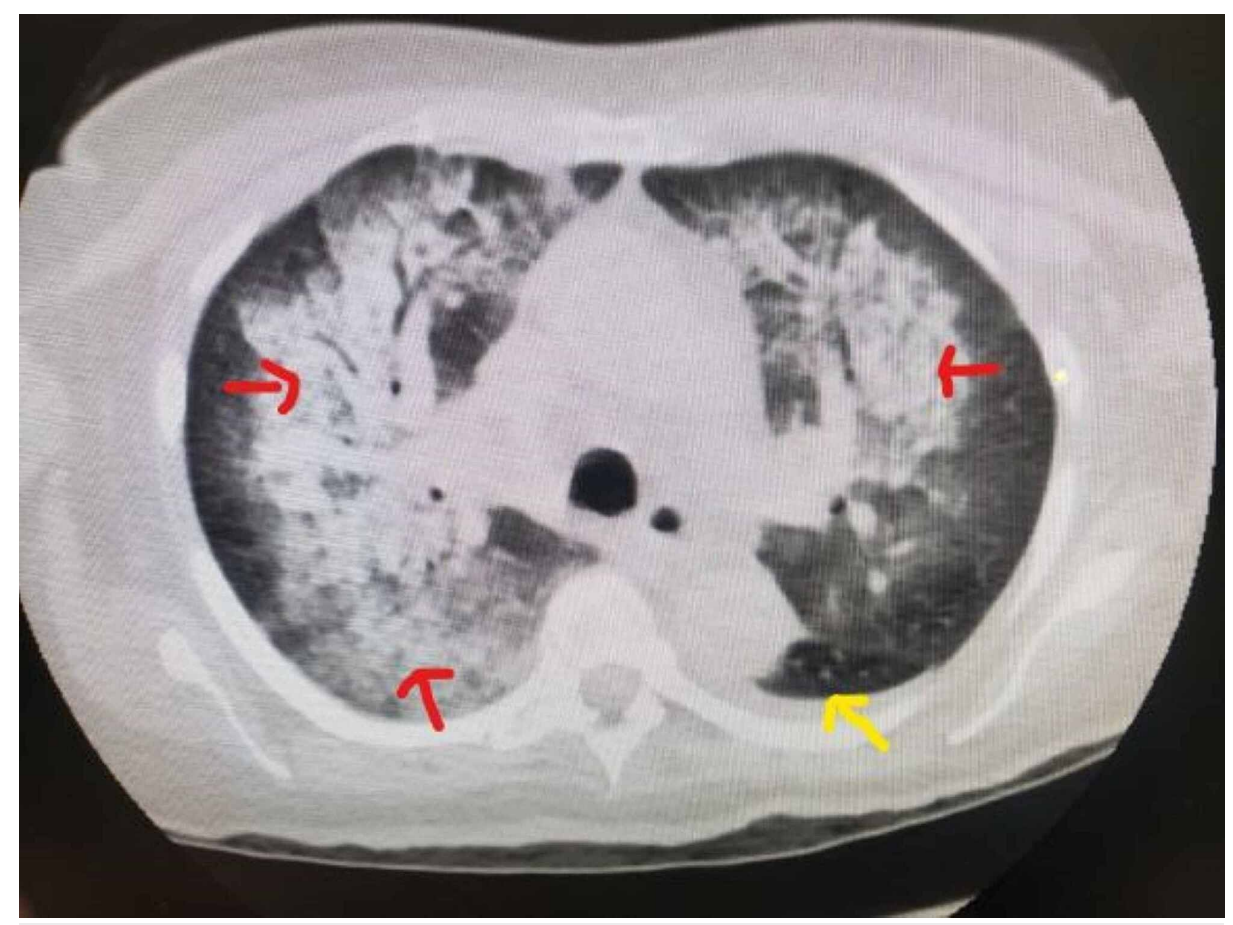

FIGURE 1: CT chest with diffuse mixed interstitial and alveolar airspace disease (red arrows) and small pleural effusion (yellow arrow)

Suspecting diffuse alveolar hemorrhage, the patient was planned for bronchoscopy, which she and the family adamantly refused. Renal biopsy was suggestive of focal glomerulosclerosis with small mesangial and subepithelial deposits suggestive of membranous lupus nephritis, the resolving phase of infection-related included in the differential. Positive multifocal red blood cell (RBC) casts consistent with the pauci-immune focal crescentic glomerulonephritis associated with ANCA (possibly hydralazine-induced).

The patient had an uneventful course in the critical care unit, completed two weeks course of antibiotics, remained on the tapering doses of prednisone (started on prednisone $60 \mathrm{mg}$ and tapering $10 \mathrm{mg}$ every week), was weaned off oxygen, BKA stump healed well, and creatinine dropped down to $4.4 \mathrm{mg} / \mathrm{l}$ and plateaued at $4.0 \mathrm{mg} / \mathrm{l}$ without dialysis. The patient was planned to be discharged to short term rehab, but the family refused and decided to take her home. The patient was discharged with improving clinical/lab status on tapering doses of prednisone and outpatient follow up with nephrology/rheumatology/surgery.

Our patient's kidney biopsy showed no definitive findings compatible with vasculitis. Our differentials remained the possibility of post-infectious/systemic lupus erythematosus (SLE)/drug-induced lupus. However, SLE remained lower on the differentials as the anti-DNA antibody and anti-Smith antibody were negative. In our clinical assessment and supporting laboratory examination (Table 2), repeat cardiolipin IgM antibody was normal. 


\section{Cureus}

\begin{tabular}{|c|c|c|}
\hline labs & Day 1 & Day 21 \\
\hline ESR (ref 0-30 mmhr) & 120 & 44 \\
\hline $\mathrm{CRP}(\mathrm{ref}<5 \mathrm{mg} / \mathrm{l})$ & 178 & 21 \\
\hline C3 (ref 90-150 mg/dl) & 53 & 92 \\
\hline C4 (ref 16-47 mg/dl) & 3 & 16 \\
\hline BUN (ref 6-20 mg/dl) & 105 & 47 \\
\hline Creatinine (ref 0.5-1.5 mg/dl) & 7.2 & 4.5 \\
\hline
\end{tabular}

\section{TABLE 2: Improving labs over days}

ESR: erythrocyte sedimentation rate. CRP: C-reactive protein, BUN: blood urea nitrogen

We believe that our patient had infectious glomerulonephritis with some element of drug-induced nephritis. Our clinical suspicion was further solidified when the patient showed improvement after below-knee amputation, a continuation of culture-specific antibiotic therapy, and discontinuation of hydralazine.

\section{Discussion}

The differential diagnosis encompassing acute glomerulonephritis is lupus nephritis, vasculitis (such as ANCA-associated vasculitis), and IgA nephropathy, to name a few. Thus, it is prudent to include the antinuclear antibodies, anti-double-stranded DNA antibodies, antineutrophil cytoplasmic antibodies (ANCA), cryoglobulins, hepatitis B and C, HIV, and anti-glomerular basement membrane antibodies in the evaluation. The specific patterns of hypocomplementemia can help narrow down the differentials like in Staphylococcus-associated or other bacterial causes of glomerulonephritis, the typical way is low C3 and normal C4 levels, lupus nephritis is usually associated with reduced levels of both C3 and C4, and mixed cryoglobulinemia is frequently associated with low C4 and normal C3 [1].

Hydralazine is known to be associated with both developing lupus and ANCA-positive vasculitis involving the kidney. The risk for the former is estimated to be $13 \%$ [2]. In patients taking hydralazine, anti-histone antibodies are present in 95\% [3]. Necrotizing glomerulonephritis is mostly implicated in drug-induced lupus with little or no immune complex deposition [4], although immune complex-mediated glomerulonephritis can occur [5]. The patients with necrotizing glomerulonephritis usually have a perinuclear anti-neutrophil cytoplasmic antibodies (P-ANCA) pattern with anti-myeloperoxidase (MPO) antibodies, plus either anti-lactoferrin or anti-elastase antibodies [4]. This combination of ANCAs is relatively specific for this form of hydralazine-induced vasculitis [6]. The most common symptoms of druginduced lupus include fever, myalgia, arthralgia, arthritis, rash, and serositis. Hematologic abnormalities, renal disease, and central nervous system involvement, although uncommon, can occur [3].

Staphylococcus-associated glomerulonephritis is rare and occurs more commonly in middle-aged to older adult patients [1]. In one single-center cohort that included 9500 kidney biopsies, the incidence of Staphylococcus-associated glomerulonephritis was found to be $0.8 \%$; the mean age was 55 years, and male to female ratio 3.5:1. Methicillin-resistant Staphylococcus aureus (MRSA) and methicillin-sensitive S. aureus (MSSA) were implicated in 59 and $27 \%$ of cases [7]. The proposed mechanisms of pathogenesis include firstly, glomerular deposition of preformed circulating immune complexes [8], and secondly, Staphylococcal antigens acting as superantigens, activating T cells, polyclonal B-cell and production of polyclonal immunoglobulin A (IgA), immunoglobulin G (IgG), and immunoglobulin M (IgM) [9].

The clinical features in adults with Staphylococcus-associated glomerulonephritis include simultaneous infection with hematuria, proteinuria of varying degrees, a rising serum creatinine, and/or edema [10]. A provisional diagnosis of Staphylococcus-associated glomerulonephritis can be made if the patient has low complement levels, endocapillary proliferation and exudative glomerulonephritis on light microscopy, C3 dominant glomerular staining on immunofluorescence microscopy, and hump-shaped subepithelial deposits on electron microscopy. Kidney biopsy is definitive, which typically shows hump-shaped subepithelial electron-dense deposits [1]. Further confirmatory is the resolution of the disease activity after eradication of the infection.

Eliminating infections is the cornerstone of the management of Staphylococcus-associated glomerulonephritis; rest involves relieving symptoms and controlling hypertension and edema by limiting salt intake and using diuretics. The role of immunosuppressants in treating Staphylococcus-associated glomerulonephritis is not well understood and is controversial in patients with an active infection, as giving 
high-dose glucocorticoids can lead to worsening of the condition or death [11]. Nasr et al. observed no correlation between glucocorticoid therapy and renal outcomes. In a series of patients with Staphylococcusassociated glomerulonephritis, $24 \%$ had complete renal function recovery, $32 \%$ had persistent renal dysfunction, and $44 \%$ progressed to end-stage renal disease [10].

We introduced steroids in our patient because there was an element of drug-induced lupus, and our experience had a positive outcome. The long-term renal prognosis in patients with Staphylococcusassociated glomerulonephritis is relatively low, which may be related to the fact that affected patients are usually older and have comorbidities such as diabetes.

\section{Conclusions}

Clinicians, when faced with acute glomerulonephritis, should keep their eyes open for any possible infection as an inciting factor and, at the same time, rule out other important causes as well. The successful management lies in early recognition, eradicating the inciting element, and prompt initiation of a specific therapy. Where the diagnosis is mixed, like in our case, clinicians must weigh the benefits versus risk of starting immunosuppressive therapy like steroids, as the steroids can act as a double-edged sword, so their judicious use is recommended.

\section{Additional Information \\ Disclosures}

Human subjects: Consent was obtained or waived by all participants in this study. Conflicts of interest: In compliance with the ICMJE uniform disclosure form, all authors declare the following: Payment/services info: All authors have declared that no financial support was received from any organization for the submitted work. Financial relationships: All authors have declared that they have no financial relationships at present or within the previous three years with any organizations that might have an interest in the submitted work. Other relationships: All authors have declared that there are no other relationships or activities that could appear to have influenced the submitted work.

\section{References}

1. Nasr SH, Radhakrishnan J, D'Agati VD: Bacterial infection-related glomerulonephritis in adults. Kidney Int. 2013, 83:792. 10.1038/ki.2012.407

2. Borchers AT, Keen CL, Gershwin ME: Drug-induced lupus. Ann N Y Acad Sci. 2007, 1108:166. 10.1016/j.tox.2004.12.025

3. Yung RL, Johnson KJ, Richardson BC: New concepts in the pathogenesis of drug-induced lupus . Lab Invest. 1995, 73:746.

4. Short AK, Lockwood CM: Antigen specificity in hydralazine associated ANCA positive systemic vasculitis . QJM. 1995, 88:775. 10.1093/oxfordjournals.qjmed.a069005

5. Shapiro KS, Pinn VW, Harrington JT, Levey AS: Immune complex glomerulonephritis in hydralazine induced SLE. Am J Kidney Dis. 1984, 3:270. 10.1016/S0272-6386(84)80044-X

6. Nässberger L, Sjöholm AG, Jonsson H, Sturfelt G, Åkesson A: Autoantibodies against neutrophil cytoplasm components in systemic lupus erythematosus and in hydralazine-induced lupus. Clin Exp Immunol. 1990, 81:380. 10.1111/j.1365-2249.1990.tb05342.x

7. Satoskar AA, Suleiman S, Ayoub I, et al.: Staphylococcus infection-associated GN - Spectrum of IgA staining and prevalence of ANCA in a single-center cohort. Clin J Am Soc Nephrol. 2017, 12:39. 10.2215/CJN.05070516

8. Nadasdy T, Hebert LA: Infection-related glomerulonephritis: understanding mechanisms. Semin Nephrol. 2011, 31:369. 10.1016/j.semnephrol.2011.06.008

9. Koyama A, Sharmin S, Sakurai H, et al.: Staphylococcus aureus cell envelope antigen is a new candidate for the induction of IgA nephropathy. Kidney Int. 2004, 66:121. 10.1111/j.1523-1755.2004.00714.x

10. Nasr SH, Fidler ME, Valeri AM, et al.: Postinfectious glomerulonephritis in the elderly. J Am Soc Nephrol. 2011, 22:187. 10.1681/ASN.2010060611

11. Rovin BH, Tang Y, Sun J, et al.: Clinical significance of fever in the systemic lupus erythematosus patient receiving steroid therapy. Kidney Int. 2005, 68:747. 10.1016/S0085-2538(15)50895-8 